\title{
Rodrigues's Formulas for Orthogonal Matrix Polynomials Satisfying Higher-Order Differential Equations
}

\author{
Antonio J. Durán
}

\section{CONTENTS}

1. Introduction

2. Proof of Lemma 1.1

3. Examples

Acknowledgments

References
We develop a method to find Rodrigues's formulas for orthogonal matrix polynomials satisfying higher-order differential equations with coefficients independent of $n$.

\section{INTRODUCTION}

The theory of orthogonal matrix polynomials starts with two papers by M. G. Krein that appeared in 1949, [Krein 49a, Krein 49b]. Each sequence of orthogonal matrix polynomials $\left(P_{n}\right)_{n}$ is associated with a weight matrix $W$ and satisfies that $P_{n}, n \geq 0$, is a matrix polynomial of degree $n$ with nonsingular leading coefficient and $\int P_{n} d W P_{m}^{*}=\Delta_{n} \delta_{n, m}$, where $\Delta_{n}, n \geq 0$, is a positive definite matrix. When $\Delta_{n}=I$, we say that the polynomials $\left(P_{n}\right)_{n}$ are orthonormal.

More than fifty years have been necessary to produce the first examples of orthogonal matrix polynomials $\left(P_{n}\right)_{n}$ satisfying second-order differential equations of the form

$$
P_{n}^{\prime \prime}(t) F_{2}(t)+P_{n}^{\prime}(t) F_{1}(t)+P_{n}(t) F_{0}=\Lambda_{n} P_{n}(t),
$$

$n=0,1, \ldots$ Here $F_{2}, F_{1}$, and $F_{0}$ are matrix polynomials (which do not depend on $n$ ) of degrees less than or equal to 2, 1, and 0, respectively (see [Durán and Grünbaum 04, Grünbaum 03, Grünbaum et al. 03], also [Durán 97]).

It is well known that in the scalar case, there are only three families of orthogonal polynomials $\left(p_{n}\right)_{n}$ with respect to a positive measure satisfying second-order differential equations of the form

$$
f_{2} p_{n}^{\prime \prime}+f_{1} p_{n}^{\prime}=\lambda_{n} p_{n}, \quad n \geq 0,
$$

where $f_{i}, i=1,2$, are polynomials of degree not larger that $i$ (independent of $n$ ), namely the classical families of Hermite, Laguerre, and Jacobi. Each one of these classical
Keywords: Orthogonal polynomials, matrix orthogonality, differential equations, Rodrigues's formulas 
families can be defined using a Rodrigues's formula:

$$
p_{n}=\left(f_{2}^{n} w\right)^{(n)} / w
$$

where $w$ is the corresponding classical weight $e^{-t^{2}}, t^{\alpha} e^{-t}$, $\alpha>-1$, or $(1+t)^{\alpha}(1-t)^{\beta}, \alpha, \beta>-1$, and $f_{2}=1, t$, and $1-t^{2}$, respectively. This kind of Rodrigues's formula also characterizes the classical families of scalar orthogonal polynomials (for a good historical account, see, for instance, [Chihara 78, Al-Salam 90]).

Instead of $(1-2)$, orthogonal matrix polynomials $P_{n}$ satisfying a differential equation like (1-1) seem to satisfy some modified Rodrigues's formula. The first instance of that modified Rodrigues's formula appeared in [Durán and Grünbaum 04]: the expression

$$
P_{n}(t)=\left[e^{-t^{2}}\left(\begin{array}{cc}
1+|a|^{2} t^{2}+|a|^{2} \frac{n}{2} & a t \\
\bar{a} t & 1
\end{array}\right)\right]^{(n)} W^{-1}(t),
$$

defines a sequence of orthogonal matrix polynomials with respect to the weight matrix

$$
W(t)=e^{-t^{2}}\left(\begin{array}{cc}
1+|a|^{2} t^{2} & a t \\
\bar{a} t & 1
\end{array}\right) .
$$

Afterward, Rodrigues's formulas of the form

$$
P_{n}(t)=\left(f_{2}^{n} \rho \xi_{n}\right)^{(n)} W^{-1},
$$

where $W=\rho Z, \xi_{n}$ are certain matrix functions, $\rho=e^{-t^{2}}$, $t^{\alpha} e^{-t}$, or $(1+t)^{\alpha}(1-t)^{\beta}$, and $f_{2}$ is equal to 1 , $t$, and $1-t^{2}$, respectively, have been found for other families of orthogonal polynomials of size $2 \times 2$ (see [Durán and Grünbaum 05a, Durán and Grünbaum 07, Durán and López-Rodríguez 07, Durán and Iglesia 08], also [Durán and Grünbaum 05b]). In all these examples, the functions $\xi_{n}$ are simple enough to make the Rodrigues's formula (1-4) useful for the explicit calculation of the sequence of orthogonal polynomials $P_{n}$ with respect to $W$.

In [Durán 10], a method to find the functions $\xi_{n}$ has been developed, and using it the first Rodrigues's formulas for families of arbitrary size $N \times N$ have been found. The method uses the following result as the main tool: under suitable conditions on the weight matrix $W$ and the differential coefficients $F_{2}, F_{1}$, and $F_{0}$ (see $(2-2),(2-$ 3 ), and $(2-4))$, we have that if for a matrix $\Lambda_{n}$, the function $\mathfrak{R}_{n}$ satisfies

$$
\begin{aligned}
& \left(\mathfrak{R}_{n} F_{2}^{*}\right)^{\prime \prime}-\left(\mathfrak{R}_{n}\left[F_{1}^{*}+n\left(F_{2}^{*}\right)^{\prime}\right]\right)^{\prime} \\
& \quad+\mathfrak{R}_{n}\left[F_{0}^{*}+n\left(F_{1}^{*}\right)^{\prime}+\left(\begin{array}{l}
n \\
2
\end{array}\right)\left(F_{2}^{*}\right)^{\prime \prime}\right]=\Lambda_{n} \mathfrak{R}_{n},
\end{aligned}
$$

then the function $\mathrm{P}_{n}=\mathfrak{R}_{n}^{(n)} W^{-1}$ satisfies

$$
\mathrm{P}_{n}^{\prime \prime}(t) F_{2}(t)+\mathrm{P}_{n}^{\prime}(t) F_{1}(t)+\mathrm{P}_{n}(t) F_{0}=\Lambda_{n} \mathrm{P}_{n}(t) .
$$

The purpose of this paper is to extend that method to find Rodrigues's formulas for orthogonal matrix polynomials satisfying higher-order differential equations. Indeed, we consider orthogonal matrix polynomials $\left(P_{n}\right)_{n}$ satisfying differential equations of the form

$$
\sum_{j=0}^{k} P_{n}^{(j)} F_{j}=\Lambda_{n} P_{n}, \quad n \geq 0,
$$

where each $F_{j}, j=0, \ldots, k$, is a matrix polynomial of degree not larger than $j$. In the scalar case, all the known examples of orthogonal polynomials satisfying higher-order differential equations of the form (1-5) are orthogonal with respect to one of the classical weights of Hermite, Laguerre, and Jacobi, or to some weights obtained by adding masses to the classical ones (at the endpoints of the orthogonality interval); see, for instance, [Littlejohn and Krall 89] or [Grünbaum and Haine 97]. The situation in the matrix case is much richer, as the examples in Section 3 of this paper show. We display there three illustrative examples of weight matrices whose entries are absolutely continuous with respect to the Lebesgue measure and supported at $(0,1), \mathbb{R}$, and $(0,+\infty)$, respectively, and are of sizes $2 \times 2$ and $3 \times 3$.

In [Durán and Iglesia 08] it was proved that under the boundary conditions

$$
\begin{aligned}
& \lim _{t \rightarrow a^{+}, b^{-}} t^{n} \sum_{i=0}^{p-1}(-1)^{k-i+p-1}\left(\begin{array}{c}
k-i \\
l
\end{array}\right)\left(F_{k-i} \cdot W\right)^{(p-1-i)} \\
& \quad=0
\end{aligned}
$$

for $p=1, \ldots, k, l=0, \ldots, k-p$, and $n \geq 0$, the differential equations

$$
\sum_{j=l}^{k}(-1)^{j}\left(\begin{array}{l}
j \\
l
\end{array}\right)\left(F_{j} W\right)^{(j-l)}=W F_{l}^{*}, \quad l=0, \ldots, k,
$$

imply that the orthonormal polynomials with respect to $W$ satisfy the differential equation (1-5) with Hermitian eigenvalues $\Lambda_{n}$.

Our method to find Rodrigues's formulas can be extended for weight matrices with a sequence of orthogonal matrix polynomials satisfying higher-order differential equations like (1-5).

Lemma 1.1. Let $F_{j}, 0 \leq j \leq k$, be matrix polynomials, $F_{j}$ of degree not larger than j. Let $W, \mathfrak{R}_{n}$ be $N \times N$ matrix functions that are respectively $k$ and $n$ times differentiable in an open set $\Omega$ of the real line. Assume that $W(t)$ is 
nonsingular for $t \in \Omega$ and that it satisfies the differential equations (1-7). Define the functions $\mathrm{P}_{n}, n \geq 1$, by

$$
\mathrm{P}_{n}=\mathfrak{R}_{n}^{(n)} W^{-1}
$$

If for a matrix $\Lambda_{n}$, the function $\mathfrak{R}_{n}$ satisfies

$$
\sum_{j=0}^{k}(-1)^{j}\left(\Re_{n} \sum_{m=j}^{k}\left(\begin{array}{c}
n \\
m-j
\end{array}\right)\left(F_{m}^{*}\right)^{(m-j)}\right)^{(j)}=\Lambda_{n} \Re_{n}
$$

then the function $\mathrm{P}_{n}$ satisfies

$$
\sum_{j=0}^{k} \mathrm{P}_{n}^{(j)}(t) F_{j}(t)=\Lambda_{n} \mathrm{P}_{n}(t)
$$

Moreover, if $\mathfrak{R}_{n}=\rho Z_{n}$, where $Z_{n}$ is a matrix polynomial and $\rho$ is equal to $e^{-t^{2}}, t^{\alpha} e^{-t}$, or $(1-t)^{\alpha}(1+t)^{\beta}$ (in this last case with $\alpha$ or $\beta$ nonintegral), then the converse is also true.

Lemma 1.1 will be proved in Section 2. Using it, we find Rodrigues's formulas for the three illustrative examples mentioned above.

To make this introduction more useful to the reader, we display in full detail one of these examples.

Consider the weight matrix

$$
W(t)=\left(\begin{array}{cc}
8 t^{3}+(1-2 t)^{2} & 2(-1+2 t)(-1+t) \\
2(-1+2 t)(-1+t) & 4(-1+t)^{2}
\end{array}\right)
$$

$0 \leq t \leq 1$. This matrix can be factored in the form

$$
W(t)=T(t)\left(\begin{array}{cc}
2 t & 0 \\
0 & 1
\end{array}\right) T^{*}(t)
$$

where

$$
T(t)=\left(\begin{array}{cc}
2 t & 1-2 t \\
0 & 2-2 t
\end{array}\right)
$$

The orthogonal matrix polynomials $\left(P_{n}\right)_{n}$ with respect to this weight matrix $W$ do not satisfy any secondorder differential equation like (1-1), but they satisfy the fourth-order differential equation

$$
\sum_{j=0}^{4} P_{n}^{(j)}(t) F_{j}(t)=\Lambda_{n} P_{n}(t)
$$

where

$$
\begin{aligned}
F_{4}(t) & =t^{2}(1-t)^{2} I, \\
F_{3}(t) & =\left(\begin{array}{cc}
2 t\left(5-12 t+7 t^{2}\right) & -t\left(3-5 t+2 t^{2}\right) \\
0 & 4 t\left(1-4 t+3 t^{2}\right)
\end{array}\right), \\
F_{2} & =\frac{1}{9}\left(\begin{array}{rr}
178-642 t+482 t^{2} & -80+223 t-134 t^{2} \\
-4+4 t & 20-252 t+340 t^{2}
\end{array}\right), \\
F_{1} & =\frac{1}{9}\left(\begin{array}{cc}
-416+520 t & 156-192 t \\
8 & -72+280 t
\end{array}\right), \\
F_{0} & =\frac{1}{9}\left(\begin{array}{cc}
64 & -16 \\
0 & 0
\end{array}\right) .
\end{aligned}
$$

Using Lemma 1.1, we find that a sequence of orthogonal polynomials $\left(P_{n}\right)_{n}$ with respect to the weight matrix $W(1-11)$ can be defined using the Rodrigues's formula

$$
P_{n}(t)=\left(t^{n}(1-t)^{n}\left(C_{0, n}+C_{1, n} t+C_{2, n} t^{2}\right) T^{*}\right)^{(n)} W^{-1},
$$

$n=1,2,3, \ldots$, where $T$ is defined by $(1-12)$ and

$$
\begin{aligned}
C_{0, n} & =\frac{9 n^{2}+27 n+16}{2}\left(\begin{array}{cc}
0 & 0 \\
n & 2(n+1)
\end{array}\right), \\
C_{1, n} & =\left(\begin{array}{cc}
n / 2 & n+2 \\
4(n+1) n & -(n+2)\left(9 n^{2}+19 n+8\right)
\end{array}\right), \\
C_{2, n} & =-\frac{9 n^{2}+27 n+16}{2}\left(\begin{array}{ll}
1 & 0 \\
0 & 0
\end{array}\right) .
\end{aligned}
$$

\section{PROOF OF LEMMA 1.1}

Before proving Lemma 1.1, we need some definitions and basic facts.

A weight matrix $W$ is an $N \times N$ matrix of measures supported in the real line satisfying the following three conditions: (1) $W(A)$ is positive semidefinite for any Borel set $A \in \mathbb{R},(2) W$ has finite moments $\int t^{n} d W(t)$ of every order $n \geq 0$, and (3) $\int P(t) d W(t) P^{*}(t)$ is nonsingular if the leading coefficient of the matrix polynomial $P$ is nonsingular. All the examples of weight matrices $W$ considered in this paper have entries with a smooth density with respect to the Lebesgue measure, and we will again write $W(t)$ for the matrix whose entries are these densities. Condition (3) above is necessary and sufficient to guarantee the existence of a sequence $\left(P_{n}\right)_{n}$ of matrix polynomials of degree $n$ orthogonal with respect to $W$ with nonsingular leading coefficient. This condition is fulfilled in particular when $W(t)$ is positive definite on an interval of the real line. 
Equations (1-1) are guaranteed by a set of differential equations for the weight matrix with respect to which the polynomials $\left(P_{n}\right)_{n}$ are orthogonal. Indeed, let $W$ be a weight matrix supported in the interval $(a, b)(a$ and $b$ finite or infinite) and assume the boundary conditions

$$
\begin{aligned}
\lim _{t \rightarrow a^{+}, b^{-}} t^{n} F_{2}(t) W(t) & =0, \\
\lim _{t \rightarrow a^{+}, b^{-}} t^{n}\left[\left(F_{2}(t) W(t)\right)^{\prime}-F_{1}(t) W(t)\right] & =0, \quad n \geq 0 .
\end{aligned}
$$

Then the equations

$$
\begin{aligned}
F_{2} W & =W F_{2}^{*}, \\
2\left(F_{2} W\right)^{\prime}-F_{1} W & =W F_{1}^{*}, \\
\left(F_{2} W\right)^{\prime \prime}-\left(F_{1} W\right)^{\prime}+F_{0} W & =W F_{0}^{*},
\end{aligned}
$$

imply that the orthonormal polynomials with respect to $W$ satisfy the second-order differential equation (11) with Hermitian eigenvalues $\Lambda_{n}$. Equations (1-7) are an extension of $(2-2),(2-3)$, and (2-4) for weight matrices whose sequences of orthogonal polynomials satisfy higher-order differential equations.

To prove Lemma 1.1, we finally need the following claim (which will be proved later).

Claim 2.1. Under the same assumptions of Lemma 1.1, we have for $l=0, \ldots, k$ that

$$
\sum_{j=l}^{k}\left(\begin{array}{l}
j \\
l
\end{array}\right)\left(W^{-1}\right)^{j-l} F_{j}=\left(\sum_{j=l}^{k}(-1)^{j}\left(\begin{array}{l}
j \\
l
\end{array}\right)\left(F_{j}^{*}\right)^{j-l}\right) W^{-1} .
$$

Proof of Lemma 1.1: Write $E_{n}=\mathfrak{R}_{n}^{(n)}$, so that $\mathrm{P}_{n}=$ $E_{n} W^{-1}$. Then it easily follows that

$$
\begin{aligned}
\sum_{j=0}^{k} \mathrm{P}_{n}^{(j)} F_{j} & =\sum_{j=0}^{k}\left(E_{n} W^{-1}\right)^{(j)} F_{j} \\
& =\sum_{j=0}^{k}\left(\sum_{l=0}^{j}\left(\begin{array}{l}
j \\
l
\end{array}\right) E_{n}^{(l)}\left(W^{-1}\right)^{(j-l)}\right) F_{j} \\
& =\sum_{l=0}^{k} E_{n}^{(l)}\left(\sum_{j=l}^{k}\left(\begin{array}{l}
j \\
l
\end{array}\right)\left(W^{-1}\right)^{(j-l)} F_{j}\right) .
\end{aligned}
$$

Using the claim, we have

$$
\sum_{j=0}^{k} \mathrm{P}_{n}^{(j)} F_{j}=\sum_{l=0}^{k} E_{n}^{(l)}\left(\sum_{j=l}^{k}(-1)^{j}\left(\begin{array}{l}
j \\
l
\end{array}\right)\left(F_{j}^{*}\right)^{j-l}\right) W^{-1} .
$$

Hence, for any matrix $\Lambda_{n}$, the function $\mathrm{P}_{n}$ satisfies the differential equation (1-10) if and only if the function
$E_{n}$ satisfies the differential equation

$$
\sum_{l=0}^{k} E_{n}^{(l)}\left(\sum_{j=l}^{k}(-1)^{j}\left(\begin{array}{l}
j \\
l
\end{array}\right)\left(F_{j}^{*}\right)^{j-l}\right)=\Lambda_{n} E_{n} .
$$

Taking into account that $E_{n}=\mathfrak{R}_{n}^{(n)}$, this equation can be written in the form

$$
\sum_{l=0}^{k} \mathfrak{R}_{n}^{(n+l)}\left(\sum_{j=l}^{k}(-1)^{j}\left(\begin{array}{l}
j \\
l
\end{array}\right)\left(F_{j}^{*}\right)^{j-l}\right)=\Lambda_{n} \mathfrak{R}_{n}^{(n)} .
$$

Now write

$$
U_{n, j}=\sum_{m=j}^{k}\left(\begin{array}{c}
n \\
m-j
\end{array}\right)\left(F_{m}^{*}\right)^{m-j}, \quad j=0, \ldots, k
$$

(where as usual we take $\left(\begin{array}{l}i \\ j\end{array}\right)=0$ if $j>i$ ). Since $F_{j}, j=$ $0, \ldots, k$, are matrix polynomials of degrees not larger than $j$, it follows that $U_{n, j}, n \geq 0, j=0, \ldots, k$, are also matrix polynomials of degrees not larger than $j$. Using this we can write

$$
\begin{aligned}
\sum_{j=0}^{k} & (-1)^{j}\left(\mathfrak{R}_{n} U_{n, j}\right)^{(j+n)} \\
& =\sum_{j=0}^{k}(-1)^{j} \sum_{l=0}^{j}\left(\begin{array}{c}
j+n \\
j-l
\end{array}\right) \Re_{n}^{(n+l)} U_{n, j}^{(j-l)} \\
& =\sum_{l=0}^{k} \mathfrak{R}_{n}^{(n+l)} \sum_{j=l}^{k}(-1)^{j}\left(\begin{array}{c}
j+n \\
j-l
\end{array}\right) U_{n, j}^{(j-l)} .
\end{aligned}
$$

Using (2-9), we have

$$
\begin{aligned}
\sum_{j=l}^{k} & (-1)^{j}\left(\begin{array}{c}
j+n \\
j-l
\end{array}\right) U_{n, j}^{(j-l)} \\
& =\sum_{j=l}^{k}(-1)^{j}\left(\begin{array}{c}
j+n \\
j-l
\end{array}\right) \sum_{m=j}^{k}\left(\begin{array}{c}
n \\
m-j
\end{array}\right)\left(F_{m}^{*}\right)^{(m-l)} \\
& =\sum_{m=l}^{k}\left(F_{m}^{*}\right)^{(m-l)} \sum_{j=l}^{m}(-1)^{j}\left(\begin{array}{c}
j+n \\
j-l
\end{array}\right)\left(\begin{array}{c}
n \\
m-j
\end{array}\right) .
\end{aligned}
$$

Taking into account now that

$$
\sum_{j=l}^{m}(-1)^{j}\left(\begin{array}{c}
j+n \\
j-l
\end{array}\right)\left(\begin{array}{c}
n \\
m-j
\end{array}\right)=(-1)^{m}\left(\begin{array}{c}
m \\
l
\end{array}\right)
$$

(which can easily be proved by induction on $n$ ), we finally have that

$$
\sum_{j=l}^{k}(-1)^{j}\left(\begin{array}{c}
j+n \\
j-l
\end{array}\right) U_{n, j}^{(j-l)}=\sum_{j=l}^{k}(-1)^{j}\left(\begin{array}{l}
j \\
l
\end{array}\right)\left(F_{j}^{*}\right)^{j-l} .
$$


This equation, (2-9), and (2-10) show that

$$
\begin{gathered}
\sum_{j=0}^{k}(-1)^{j}\left(\mathfrak{R}_{n} \sum_{m=j}^{k}\left(\begin{array}{c}
n \\
m-j
\end{array}\right)\left(F_{m}^{*}\right)^{m-j}\right)^{(j+n)} \\
=\sum_{l=0}^{k} \mathfrak{R}_{n}^{(n+l)}\left(\sum_{j=l}^{k}\left(\begin{array}{l}
j \\
l
\end{array}\right)(-1)^{j}\left(F_{j}^{*}\right)^{(j-l)}\right) .
\end{gathered}
$$

Hence, we can rewrite $(2-8)$ in the form

$$
\begin{aligned}
& {\left[\sum_{j=0}^{k}(-1)^{j}\left(\Re_{n} \sum_{m=j}^{k}\left(\begin{array}{c}
n \\
m-j
\end{array}\right)\left(F_{m}^{*}\right)^{m-j}\right)^{(j)}-\Lambda_{n} \Re_{n}\right]_{(2-11)}^{(n)}} \\
& \quad=0 .
\end{aligned}
$$

This finishes the proof of the first part of Lemma 1, since the function $\mathrm{P}_{n}$ satisfies the differential equation (1-10) if and only if the function $\mathfrak{R}_{n}$ satisfies the differential equation $(2-8)$.

Assume now that $\mathfrak{R}_{n}=\rho Z_{n}$, where $Z_{n}$ is a matrix polynomial and $\rho$ equals $e^{-t^{2}}, t^{\alpha} e^{-t}$, or $(1-t)^{\alpha}(1+t)^{\beta}$ (in this last case with $\alpha$ or $\beta$ nonintegral). Since $\rho$ and 1 are linearly independent in the linear space of polynomials, we have that $(2-11)$ is equivalent to

$$
\sum_{j=0}^{k}(-1)^{j}\left(\Re_{n} \sum_{m=j}^{k}\left(\begin{array}{c}
n \\
m-j
\end{array}\right)\left(F_{m}^{*}\right)^{m-j}\right)^{(j)}-\Lambda_{n} \Re_{n}=0 .
$$

This proves the second part of Lemma 1.1.

We now prove the claim.

Proof of Claim 2.1: To do so, we use the formula

$$
\left(W^{-1}\right)^{(l)}=-W^{-1} \sum_{m=1}^{l}\left(\begin{array}{c}
l \\
m
\end{array}\right) W^{(m)}\left(W^{-1}\right)^{(l-m)},
$$

which can be easily deduced by differentiating the identity $W W^{-1}=I$.

We proceed by induction on $l$. For $l=k$, formula $(2-5)$ just reduces to $l=k$ in $(1-7)$.

We now assume that $(2-5)$ holds for $k, k-1, \ldots, l$ and prove it for $l-1$. Using $(2-12)$, we can write

$$
\begin{gathered}
\sum_{j=l-1}^{k}\left(\begin{array}{c}
j \\
l-1
\end{array}\right)\left(W^{-1}\right)^{j-l+1} F_{j} \\
=W^{-1} F_{l-1}-W^{-1} \sum_{j=l}^{k}\left(\begin{array}{c}
j \\
l-1
\end{array}\right) \sum_{m=1}^{j-l+1}\left(\begin{array}{c}
j-l+1 \\
m
\end{array}\right) \\
\times W^{(m)}\left(W^{-1}\right)^{(j-l+1-m)} F_{j} .
\end{gathered}
$$

Taking into account that

$$
\left(\begin{array}{c}
j-l+1 \\
m
\end{array}\right)\left(\begin{array}{c}
j \\
l-1
\end{array}\right)=\left(\begin{array}{c}
j \\
l+m-1
\end{array}\right)\left(\begin{array}{c}
l+m-1 \\
m
\end{array}\right)
$$

we have

$$
\begin{aligned}
& \sum_{j=l-1}^{k}\left(\begin{array}{c}
j \\
l-1
\end{array}\right)\left(W^{-1}\right)^{j-l+1} F_{j} \\
& =W^{-1} F_{l-1} \\
& \quad-W^{-1} \sum_{m=1}^{k-l+1}\left(\begin{array}{c}
l+m-1 \\
m
\end{array}\right) W^{(m)} \\
& \quad \times \sum_{j=l+m-1}^{k}\left(\begin{array}{c}
j \\
l+m-1
\end{array}\right)\left(W^{-1}\right)^{(j-l+1-m)} F_{j},
\end{aligned}
$$

which using the induction hypothesis can be written in the form

$$
\begin{aligned}
& \sum_{j=l-1}^{k}\left(\begin{array}{c}
j \\
l-1
\end{array}\right)\left(W^{-1}\right)^{j-l+1} F_{j} \\
& =W^{-1} F_{l-1} \\
& \quad-W^{-1} \sum_{m=1}^{k-l+1}\left(\begin{array}{c}
l+m-1 \\
m
\end{array}\right) W^{(m)} \\
& \quad \times \sum_{j=l+m-1}^{k}(-1)^{j}\left(\begin{array}{c}
j \\
l+m-1
\end{array}\right)\left(F_{j}^{*}\right)^{(j-l+1-m)} W^{-1} .
\end{aligned}
$$

On the other hand, we can rewrite equation (1-7), for $l-1$, as follows:

$$
\begin{aligned}
F_{l} W= & \sum_{j=l-1}^{k}(-1)^{j}\left(\begin{array}{c}
j \\
l-1
\end{array}\right)\left(W F_{j}^{*}\right)^{(j-l+1)} \\
= & \sum_{j=l-1}^{k}(-1)^{j}\left(\begin{array}{c}
j \\
l-1
\end{array}\right) \sum_{m=0}^{j-l+1}\left(\begin{array}{c}
j-l+1 \\
m
\end{array}\right) W^{(m)} \\
& \times\left(F_{j}^{*}\right)^{(j-l+1-m)} \\
= & \sum_{m=0}^{k-l+1} W^{(m)} \sum_{j=l+m-1}^{k}(-1)^{j}\left(\begin{array}{c}
j \\
l-1
\end{array}\right)\left(\begin{array}{c}
j-l+1 \\
m
\end{array}\right) \\
& \times\left(F_{j}^{*}\right)^{(j-l+1-m)} .
\end{aligned}
$$

Using (2-13), this gives

$$
\begin{aligned}
F_{l} W & -W \sum_{j=l-1}^{k}(-1)^{j}\left(\begin{array}{c}
j \\
l-1
\end{array}\right)\left(F_{j}^{*}\right)^{(j-l+1)} \\
= & \sum_{m=1}^{k-l+1}\left(\begin{array}{c}
l+m-1 \\
m
\end{array}\right) W^{(m)} \sum_{j=l+m-1}^{k}(-1)^{j}\left(\begin{array}{c}
j \\
l+m-1
\end{array}\right) \\
& \times\left(F_{j}^{*}\right)^{(j-l+1-m)} .
\end{aligned}
$$


Inserting this formula into $(2-14)$, we have

$$
\begin{aligned}
\sum_{j=l-1}^{k}\left(\begin{array}{c}
j \\
l-1
\end{array}\right)\left(W^{-1}\right)^{j-l+1} F_{j} \\
=W^{-1} F_{l-1}-W^{-1} F_{l}+\sum_{j=l-1}^{k}(-1)^{j}\left(\begin{array}{c}
j \\
l-1
\end{array}\right) \\
\quad \times\left(F_{j}^{*}\right)^{(j-l+1)} W^{-1} \\
=\sum_{j=l-1}^{k}(-1)^{j}\left(\begin{array}{c}
j \\
l-1
\end{array}\right)\left(F_{j}^{*}\right)^{(j-l+1)} W^{-1},
\end{aligned}
$$

and the proof of the claim is finished.

\section{EXAMPLES}

We now use Lemma 1.1 to find Rodrigues's formulas for some examples of orthogonal matrix polynomials satisfying higher-order differential equations of the form (1-5).

Our strategy of using Lemma 1.1 to find Rodrigues's formulas is as follows: We assume that we have a weight matrix of the form $\rho Z$, with $\rho$ a scalar classical weight (Hermite, Laguerre, or Jacobi), satisfying equations (1-7) for certain coefficients $F_{j}, j=0, \ldots, k$. Under the boundary conditions $(1-6)$, we have that any sequence $\left(P_{n}\right)_{n}$ of orthogonal polynomials with respect to $W$ satisfies a higher-order differential equation like (1-5) for a suitable choice of eigenvalues $\left(\Lambda_{n}\right)_{n}$. The sequence of eigenvalues $\left(\Lambda_{n}\right)_{n}$ corresponding to the sequence $\left(\hat{P}_{n}\right)_{n}$ of monic orthogonal polynomials is

$$
\Lambda_{n}=\sum_{j=0}^{k}\left(\begin{array}{l}
n \\
j
\end{array}\right)\left(F_{j}\right)^{(j)}
$$

Of course, we can choose another sequence of eigenvalues; to do so, it is enough to take into account that for any sequence of nonsingular matrices $\left(N_{n}\right)_{n}$, the polynomials $P_{n}=N_{n} \hat{P}_{n}, n \geq 0$, are also orthogonal with respect to $W$, and the sequence $\left(\Lambda_{n}\right)_{n}$ of eigenvalues corresponding to $\left(P_{n}\right)_{n}$ is then

$$
\Lambda_{n}=N_{n}\left(\sum_{j=0}^{k}\left(\begin{array}{l}
n \\
j
\end{array}\right)\left(F_{j}\right)^{(j)}\right) N_{n}^{-1} .
$$

Assume also that $Z$ can be factored as $Z=T D T^{*}$, where $T$ is a matrix polynomial and $D$ is a diagonal matrix whose diagonal entries are integer powers of $t$. We then look for solutions $\mathfrak{R}_{n}, n \geq 1$, of the differential equations (1-9) of the form $\mathfrak{R}_{n}=\rho f_{2}^{n} \xi_{n} T^{*}$, where $f_{2}=1$ for $\rho=e^{-t^{2}}, f_{2}=t$ for $\rho=t^{\alpha} e^{-t}$, and $f_{2}=t(1-t)$ for $\rho=t^{\alpha}(1-t)^{\beta}$. The illustrative examples we display in this section show that the functions $\xi_{n}, n \geq 1$, likely can be chosen to be polynomials of degree independent of $n$.

We display three examples corresponding to weight matrices supported in the compact interval $(0,1)$, the half-line $(0,+\infty)$, and the whole real line $\mathbb{R}$, respectively.

Example 3.1. We first consider the weight matrix $W$ defined by (1-11). The weight matrix $W$ does not satisfy any differential equation of the form (2-4) for any sets of coefficients $F_{2}, F_{1}, F_{0}$. Moreover, if we take any sequence of polynomials $\left(P_{n}\right)_{n}$ orthogonal with respect to this weight matrix $W$, they do not satisfy any secondorder differential equation like (1-1).

On the other hand, the weight matrix $W$ satisfies the set of equations

$$
\sum_{j=l}^{4}(-1)^{j}\left(\begin{array}{l}
j \\
l
\end{array}\right)\left(F_{j} W\right)^{(j-l)}=W F_{l}^{*}, \quad l=0, \ldots, 4,
$$

where $F_{i}, i=0,1,2,3,4$, are given by (1-13). Actually, there is another set of coefficients $G_{i}, i=0,1,2,3,4\left(F_{i}\right.$ and $G_{i}$ linearly independent) such that $W$ satisfies

$$
\sum_{j=l}^{4}(-1)^{j}\left(\begin{array}{l}
j \\
l
\end{array}\right)\left(G_{j} W\right)^{(j-l)}=W G_{l}^{*}, \quad l=0, \ldots, 4,
$$

namely

$$
\begin{aligned}
G 4 & =\frac{1-t}{4}\left(\begin{array}{ll}
0 & 9 t^{2}(1-2 t) \\
0 & 18 t^{2}(1-t)
\end{array}\right) \\
G 3 & =\frac{1}{4}\left(\begin{array}{cc}
2(-1+2 t)(-1+t) & -1+40 t-220 t^{2}+208 t^{3} \\
4(-1+t)^{2} & 2(-1+t)\left(1-38 t+108 t^{2}\right)
\end{array}\right), \\
G 2 & =\frac{1}{18}\left(\begin{array}{cc}
-80+114 t-16 t^{2} & 121-1772 t+2740 t^{2} \\
-106(1-t) & 215-2322 t+3052 t^{2}
\end{array}\right), \\
G 1 & =\frac{1}{9}\left(\begin{array}{cc}
68-40 t & -264+948 t \\
52 & -324+1244 t
\end{array}\right) \\
G 0 & =\frac{1}{9}\left(\begin{array}{cc}
-160 & 40 \\
0 & 0
\end{array}\right) .
\end{aligned}
$$

Since the boundary conditions $(1-6)$ are satisfied, we have that the monic orthogonal polynomials $\left(\hat{P}_{n}\right)_{n}$ with respect to $W$ satisfy the fourth-order differential equation

$$
\sum_{j=0}^{4} \hat{P}_{n}^{(j)}(t) F_{j}(t)=\Gamma_{n} \hat{P}_{n}(t)
$$

where $\Gamma_{n}=\sum_{j=0}^{4}\left(\begin{array}{c}n \\ j\end{array}\right) F_{j}^{(j)}(t)$. This gives

$$
\Gamma_{n}=\frac{1}{9}\left(\begin{array}{cc}
9 n^{4}+72 n^{3}+203 n^{2}+236 n+64 & -18 n^{3}-80 n^{2}-94 n-16 \\
0 & n\left(9 n^{3}+54 n^{2}+115 n+102\right)
\end{array}\right) .
$$


To simplify the computations, we normalize these eigenvalues using the matrix

$$
N_{n}=\left(\begin{array}{cc}
n+2 & 0 \\
0 & 9 n^{3}+40 n^{2}+47 n+8
\end{array}\right) .
$$

Hence, we define

$$
\begin{aligned}
\Lambda_{n} & =N_{n} \Gamma_{n} N_{n}^{-1} \\
& =\frac{1}{9}\left(\begin{array}{cc}
9 n^{4}+72 n^{3}+203 n^{2}+236 n+64 & -2 n-4 \\
0 & n\left(9 n^{3}+54 n^{2}+115 n+102\right)
\end{array}\right) .
\end{aligned}
$$

We now solve the associated fourth-order differential equation

$$
\sum_{j=0}^{4}(-1)^{j}\left(\Re_{n} \sum_{m=j}^{k}\left(\begin{array}{c}
n \\
m-j
\end{array}\right)\left(F_{m}^{*}\right)^{(m-j)}\right)^{(j)}=\Lambda_{n} \Re_{n} .
$$

Taking into account our strategy, we look for solutions $\mathfrak{R}_{n}$ of the form

$$
\Re_{n}(t)=t^{n}(1-t)^{n}\left(C_{0, n}+C_{1, n} t+C_{2, n} t^{2}\right) T^{*},
$$

where $T$ is the polynomial defined by (1-12). In doing so, we find the matrices $C_{j, n}, j=0,1,2$, given by (114). The polynomial $\mathfrak{R}_{n}$ defined by these matrices then satisfies the differential equation (3-3).

Using Lemma 1.1, we deduce that the matrix functions defined by

$$
\mathrm{P}_{n}=\mathfrak{R}_{n}^{(n)} W^{-1}, \quad n \geq 1,
$$

satisfy the fourth-order differential equation

$$
\sum_{j=0}^{4} \mathrm{P}_{n}^{(j)}(t) F_{j}(t)=\Lambda_{n} \mathrm{P}_{n}(t) .
$$

Once we have found the functions $\mathfrak{R}_{n}$, we have to prove that (3-4) defines a sequence of orthogonal matrix polynomials with respect to $W$.

First of all, we prove that the function $\mathrm{P}_{n}, n \geq 1$, is a polynomial of degree $n$. This can be done in different ways. One can just proceed by performing a direct computation (in a similar way to the proof of [Durán and Grünbaum 05a, Theorem 3.1 ] or [Durán and LópezRodríguez 07, Theorems 2.1 and 2.2 ]).

But we can also prove that $\mathrm{P}_{n}$ is a polynomial of degree $n$ using the differential equation (3-5). Indeed, since

$$
W^{-1}(t)=\frac{1}{t^{3}}\left(\begin{array}{cc}
\frac{1}{8} & -\frac{-1+2 t}{16(-1+t)} \\
-\frac{-1+2 t}{16(-1+t)} & \frac{1-4 t+4 t^{2}+8 t^{3}}{32(1-t)^{2}}
\end{array}\right),
$$

we deduce that (see $(3-4))$

$$
\mathrm{P}_{n}(t)=\sum_{j=-3}^{n+1} D_{j} t^{j}+\frac{E_{-1}}{1-t}+\frac{E_{-2}}{(1-t)^{2}} .
$$

We have to prove that $D_{-3}=D_{-2}=D_{-1}=D_{n+1}=$ $E_{-1}=E_{-2}=0$.

To do so, we proceed by reductio ad absurdum. Assume first that $\mathrm{P}_{n}$ has a pole at $t=0$ of order $r>0$. This means that $D_{-r} \neq 0,0<r \leq 3$. If we compare the coefficient of $1 / t^{r}$ on both sides of $(3-5)$, we find that

$$
D_{-r} \Gamma_{-r}-\Lambda_{n} D_{-r}=0 .
$$

The matrices $\Gamma_{n}$ and $\Lambda_{n}$ (see (3-1) and (3-2)) have the same eigenvalues, namely

$$
\begin{aligned}
& \lambda_{n, 1}=n^{4}+8 n^{3}+\frac{203 n^{2}+236 n+64}{9}, \\
& \lambda_{n, 2}=n\left(n^{3}+6 n^{3}+\frac{115 n+102}{9}\right) .
\end{aligned}
$$

It is easy to see that

$$
\lambda_{-r, i} \leq 0<\lambda_{n, i}, \quad r=1,2,3, n \geq 1, \text { and } i=1,2,
$$

and

$$
0<\lambda_{n, 2}<\lambda_{n, 1}<\lambda_{n+1,2}, \quad \text { for } n \geq 1 \text {. }
$$

Then (3.7) shows that the matrices $\Gamma_{-r}, 0<r \leq 3$, and $\Lambda_{n}, n \geq 1$, have no common eigenvalue. We then deduce from (3-6) that $D_{-r}=0$ (see [Gantmacher 60, p. 225]), which is a contradiction. Hence $\mathrm{P}_{n}$ has no pole at $t=0$.

In the same way, it can be proved that $\mathrm{P}_{n}$ has no pole at $t=1$, and that it is a polynomial of degree at most $n$ (using $(3-8))$.

Once we have proved that $\mathrm{P}_{n}$ is a polynomial of degree at most $n$, integration by parts shows that

$$
\int_{0}^{1} \mathrm{P}_{n}(t) W(t) t^{k} d t=0, \quad k=0, \ldots, n-1 .
$$

In particular, this implies that $\mathrm{P}_{n}$ has degree $n$, and that $\mathrm{P}_{n}=C \hat{P}_{n}$, where $\hat{P}_{n}$ is the $n$th monic orthogonal polynomial and $C$ the leading coefficient of $\mathrm{P}_{n}$. Since

$$
\int_{0}^{1} \mathrm{P}_{n}(t) W(t) t^{n} d t=C \int_{0}^{1} \hat{P}_{n}(t) W(t) \hat{P}_{n}(t) d t
$$

and $\int_{0}^{1} \hat{P}_{n}(t) W(t) \hat{P}_{n}(t) d t$ is positive definite, it follows that $C$ is nonsingular if and only if $\int_{0}^{1} \mathrm{P}_{n}(t) W(t) t^{n} d t$ is nonsingular. Integration by parts gives

$$
\int_{0}^{1} \mathrm{P}_{n}(t) W(t) t^{n} d t=(-1)^{n} n ! \int_{0}^{1} \mathfrak{R}_{n}(t) d t .
$$


A direct calculation shows that

$$
\begin{aligned}
& \operatorname{det}\left(\int_{0}^{1} \mathfrak{R}_{n}(t) d t\right) \\
& =-\frac{(n+1) !^{2} \pi(n+3)\left(9 n^{2}+37 n+36\right)\left(9 n^{2}+27 n+16\right)}{64(16)^{n} \Gamma^{2}(n+5 / 2)} \\
& \neq 0,
\end{aligned}
$$

from which we deduce that $\int_{0}^{1} \mathrm{P}_{n}(t) W(t) t^{n} d t$ is nonsingular.

We have then proved that the function $\mathrm{P}_{n}$ defined by $(3-4)$ is a polynomial of degree $n$ with nonsingular leading coefficient and orthogonal to $t^{k}, k=0, \ldots, n-1$, with respect to $W$. Hence $\left(\mathrm{P}_{n}\right)_{n}$, defined by $(3-4)$, is a sequence of orthogonal polynomials with respect to $W$.

Example 3.2. We now consider the weight matrix

$$
W(t)=e^{-t^{2}} e^{A t} e^{A^{*} t},
$$

where $A$ is the $3 \times 3$ nilpotent matrix

$$
A=\left(\begin{array}{lll}
0 & a & c \\
0 & 0 & b \\
0 & 0 & 0
\end{array}\right)
$$

and $a, b$, and $c$ are real numbers. Since $A$ is nilpotent of order at most 3 , the matrix function $e^{A t}$ is actually a matrix polynomial of degree at most 3 . An easy calculation shows that $W(t)=e^{-t^{2}} Z(t)$, where $Z$ is the following matrix polynomial:

$Z(t)=\left(\begin{array}{ccc}1+a^{2} t^{2}+c^{2} t^{2}+a b c t^{3}+\frac{a^{2} b^{2} t^{4}}{4} & a t+b c t^{2}+\frac{a b^{2} t^{3}}{2} & c t+\frac{a b t^{2}}{2} \\ a t+b c t^{2}+\frac{a b^{2} t^{3}}{2} & 1+b^{2} t^{2} & b t \\ c t+\frac{a b t^{2}}{2} & b t & 1\end{array}\right)$.

It was proved in [Durán and Grünbaum 04] that for $c=0$, this weight matrix satisfies equations $(2-2),(2-3)$, and (2-4) for

$$
F_{2}=I, \quad F_{1}=2 A-2 t I, \quad F_{0}=\left(\begin{array}{ccc}
-4 & 0 & a b \\
0 & -2 & 0 \\
0 & 0 & 0
\end{array}\right)
$$

and then any sequence of polynomials $\left(P_{n}\right)_{n}$ orthogonal with respect to this weight matrix $W$ satisfies the secondorder differential equations (1-1).

But when $c \neq 0$, the weight matrix $W$ does not satisfy any differential equation of the form (2-4) for any sets of coefficients $F_{2}, F_{1}, F_{0}$.
On the other hand, the weight matrix $W$ satisfies the set of equations

$$
\sum_{j=l}^{4}(-1)^{j}\left(\begin{array}{l}
j \\
l
\end{array}\right)\left(F_{j} W\right)^{(j-l)}=W F_{l}^{*}, \quad l=0, \ldots, 4,
$$

for certain coefficients $F_{i}, i=0,1,2,3,4$ (actually, there are five linearly independent sets of coefficients satisfying $(3-12))$.

For concreteness we display a set of coefficients $F_{4}$, $F_{3}, F_{2}, F_{1}$, and $F_{0}$ for the particular values $a=1, b=2$, $c=-1$ :

$$
\begin{aligned}
F_{4}(t) & =\left(\begin{array}{ccc}
7 & -t & 2 t \\
0 & 6 & -2 t \\
0 & 0 & 5
\end{array}\right), \\
F_{3}(t) & =\left(\begin{array}{ccc}
-24 t & 20-2 t+2 t^{2} & -20-2 t \\
4 & -24 t & 38+4 t+4 t^{2} \\
0 & 2 & -24 t
\end{array}\right), \\
F_{2}(t) & =\frac{1}{3}\left(\begin{array}{ccc}
-334-8 t+60 t^{2} & -26-70 t+4 t^{2} & 162+40 t-28 t^{2} \\
-8-24 t & -252-24 t+72 t^{2} & -16-156 t+32 t^{2} \\
0 & -16-12 t & -158+32 t+84 t^{2}
\end{array}\right), \\
F_{1}(t) & =\frac{1}{3}\left(\begin{array}{ccc}
-16+472 t & -348+88 t & 284-172 t \\
-136 & -64+432 t & -540+56 t \\
0 & -80 & 416 t
\end{array}\right), \\
F_{0}(t) & =\frac{1}{3}\left(\begin{array}{ccc}
384 & 128 & -224 \\
64 & 208 & 64 \\
0 & 32 & 0
\end{array}\right) .
\end{aligned}
$$

We can apply our strategy to $W$ (as in the previous example) and find that a sequence of orthogonal polynomials $\left(P_{n}\right)_{n}$ with respect to the weight matrix $W$ can be defined using the following Rodrigues's formula:

$$
P_{n}(t)=\left(e^{-t^{2}}\left(C_{0, n}+C_{1, n} t+C_{2, n} t^{2}\right) T^{*}\right)^{(n)} W^{-1},
$$

$n=1,2,3, \ldots$, where

$$
T(t)=e^{A t},
$$

and $C_{0, n}, C_{1, n}$, and $C_{2, n}$ are the matrices:

$$
\begin{aligned}
C_{0, n} & =\left(\begin{array}{ccc}
\frac{n a^{2} b^{2}(n+1)}{16}+\frac{n\left(a^{2}+c^{2}\right)}{2}+1 & \frac{n b c\left(n a^{2}-2 a^{2}+8\right)}{4\left(n a^{2}+4\right)} & 0 \\
n b c / 2 & \frac{n b^{2}\left[a^{2}(n-1)+8\right]+16}{4\left(n a^{2}+4\right)} & 0 \\
0 & \frac{-n a c}{n a^{2}+4} & 1
\end{array}\right), \\
C_{1, n} & =\frac{1}{4}\left(\begin{array}{ccc}
c a b n & \left(n b^{2}+4\right) a & 4 c \\
0 & 0 & 4 b \\
0 & 0 & 0
\end{array}\right), \\
C_{2, n} & =\frac{1}{2}\left(\begin{array}{ccc}
0 & 0 & a b \\
0 & 0 & 0 \\
0 & 0 & 0
\end{array}\right) .
\end{aligned}
$$

The case in which $c=0$ and $a$ and $b$ satisfy

$$
a^{2} b^{2}-4 b^{2}+4 a^{2}=0
$$

is especially interesting. The weight matrix $W$ then satisfies equations $(2-2),(2-3)$, and $(2-4)$ for linearly independent sets of coefficients $F_{2}, F_{1}$, and $F_{0}$ (see [Durán 09]), and the Rodrigues's formula (3-13) can be written 
in a rather compact form:

$$
P_{n}(t)=\left(e^{-t^{2}} T D_{n} T^{*}\right)^{(n)} W^{-1}, \quad n=1,2,3, \ldots
$$

where $D_{n}$ is the diagonal matrix

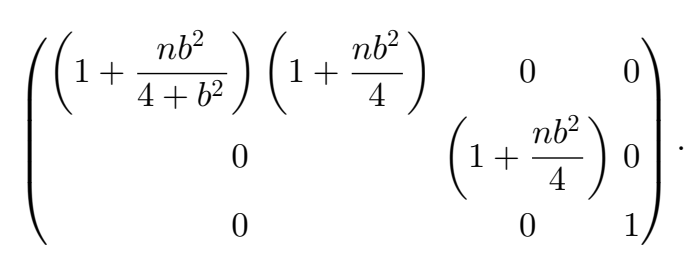

(See [Durán 10] for an extension of this Rodrigues's formula to arbitrary size $N \times N$.)

This example, together with other symbolic computational evidence, allows us to conjecture the following example of arbitrary size. Let $A$ be an $N \times N$ nilpotent matrix and consider the weight matrix $W_{A}(t)=e^{-t^{2}} e^{A t} e^{A^{*} t}$. Only when $A$ is unitarily equivalent to the nilpotent matrix

$$
\left(\begin{array}{ccccc}
0 & v_{1} & 0 & \cdots & 0 \\
0 & 0 & v_{2} & \cdots & 0 \\
\vdots & \vdots & \vdots & \ddots & \vdots \\
0 & 0 & 0 & \cdots & v_{N-1} \\
0 & 0 & 0 & \cdots & 0
\end{array}\right)
$$

do the orthogonal polynomials with respect to $W_{A}$ satisfy a second-order differential equation like (1-1) with $F_{2}=I$ (see [Durán and Grünbaum 04] and [Durán and Grünbaum 05c]). Our conjecture is that when $A$ is nilpotent, the orthogonal polynomials with respect to $W_{A}$ satisfy differential equations like (1-5) with order $k$, $k \leq 2^{N-1}$.

Example 3.3. Our last example is the weight matrix

$$
W(t)=e^{-t}\left(\begin{array}{ccc}
2 t^{3}+t^{4} & t^{2}+2 t^{3} & t^{2} \\
t^{2}+2 t^{3} & t+4 t^{2} & 2 t \\
t^{2} & 2 t & 1
\end{array}\right), \quad t \geq 0
$$

As in the previous examples, the weight matrix $W$ does not satisfy any differential equation of the form (2-4) for any sets of coefficients $F_{2}, F_{1}, F_{0}$, but it does satisfy the set of equations

$$
\sum_{j=l}^{4}(-1)^{j}\left(\begin{array}{l}
j \\
l
\end{array}\right)\left(F_{j} W\right)^{(j-l)}=W F_{l}^{*}, \quad l=0, \ldots, 4
$$

where

$$
\begin{aligned}
& F_{4}(t)=\frac{1}{1578}\left(\begin{array}{ccc}
-2856 t^{2} & 1428 t^{3} & 0 \\
-977 t & 526 t^{2} & -75 t^{3} \\
0 & 0 & 0
\end{array}\right) \text {, } \\
& F_{3}(t)=\frac{1}{4734} \\
& \times\left(\begin{array}{ccc}
-79818 t+8596 t^{2} & 31116 t^{2}-4298 t^{3} & 30396 t^{3} \\
-11724-11263 t & 3606 t+5519 t^{2} & 8737 t^{2}+225 t^{3} \\
-8585 & 4360 t & -135 t^{2}
\end{array}\right), \\
& F_{2}(t)=\frac{1}{4734} \\
& \times\left(\begin{array}{ccc}
-147912+60924 t & 9924 t-37924 t^{2} & 234904 t^{2} \\
-40389+14250 t & -8118+1068 t-7125 t^{2} & 33136 t+51080 t^{2} \\
8631 & -8450-4383 t & 27527 t+135 t^{2}
\end{array}\right), \\
& F_{1}(t)=\frac{1}{4734}\left(\begin{array}{ccc}
79620+2072 t & -86580-78184 t & 369940 t \\
35228 & -44178-26147 t & 8450+141833 t \\
-18 & -6523+9 t & 10627+2419 t
\end{array}\right) \text {, } \\
& F_{0}(t)=\frac{1}{4734}\left(\begin{array}{ccc}
1849 & -31976 & 59960 \\
-70 & -13936 & 27823 \\
0 & -21 & 0
\end{array}\right) \text {. }
\end{aligned}
$$

(Actually, there are two linearly independent sets of coefficients satisfying (3-14).)

This weight matrix can be factored in the form $W(t)=T(t)\left(\begin{array}{ccc}t^{-1} & 0 & 0 \\ 0 & t^{-1} & 0 \\ 0 & 0 & 1\end{array}\right) T^{*}(t)$, where

$$
T(t)=\left(\begin{array}{ccc}
t^{2} & t^{2} & t^{2} \\
0 & t & 2 t \\
0 & 0 & 1
\end{array}\right)
$$

We can then apply our strategy to $W$ and find that a sequence of orthogonal polynomials $\left(P_{n}\right)_{n}$ with respect to the weight matrix $W$ can be defined using the following Rodrigues's formula:

$$
P_{n}(t)=\left(e^{-t} t^{n}\left(C_{0, n}+C_{1, n} t+C_{2, n} t^{2}\right) T^{*}\right)^{(n)} W^{-1},
$$

$n=1,2,3, \ldots$, where $C_{0, n}, C_{1, n}$, and $C_{2, n}$ are the matrices

$$
\begin{aligned}
& C_{0, n}= \\
& \left(\begin{array}{ccc}
-n(13 n+3) & 13 n^{2}+41 n-6 & -12 n-12 \\
2\left(5 n^{2}+13 n+2\right) n & -18 n^{3}-50 n^{2}-44 n-8 & 4(2 n+3)(n+1) n \\
(n-1) n & n(2 n+1)(n-1) & -2(n+1)\left(n^{2}+n+1\right)
\end{array}\right), \\
& C_{1, n}=\left(\begin{array}{ccc}
29 & 58 n+29 & -12 n-12 \\
0 & 0 & -2\left(23 n^{3}+63 n^{2}+46 n+8\right) /(4 n+1) \\
0 & 0 & n^{2}-n
\end{array}\right), \\
& C_{2, n}=\left(\begin{array}{ccc}
0 & 0 & 29 \\
0 & 0 & 0 \\
0 & 0 & 0
\end{array}\right) .
\end{aligned}
$$

\section{ACKNOWLEDGMENTS}

This work was partially supported by D.G.E.S, ref. MTM2009-12740-C03-02, FQM-262, FQM-4643 (Junta de Andalucía). 


\section{REFERENCES}

[Al-Salam 90] W. Al Salam. "Characterization Theorems for Orthogonal Polynomials." in Orthogonal Polynomials: Theory and Practice, edited by P. Nevai and M. Ismail, pp. 1-24. Dordrecht: Kluwer Academic Pub., 1990.

[Chihara 78] T. Chihara. An Introduction to Orthogonal Polynomials. New York: Gordon and Breach Science Publishers, 1978.

[Durán 97] A. J. Durán. "Matrix Inner Product Having a Matrix Symmetric Second Order Differential Operator." Rocky Mount. J. Math. 27 (1997), 585600 .

[Durán 09] A. J. Durán. "A Method to Find Weight Matrices Having Symmetric Second Order Differential Operators with Matrix Leading Coefficient." Constr. Approx. 29 (2009), 181-205.

[Durán 10] A. J. Durán. "Rodrigues's Formulas for Orthogonal Matrix Polynomials Satisfying Second Order Differential Equations." International Math. Research Notices 2010 (2010), 824-855

[Durán and Grünbaum 04] A. J. Durán and F. A. Grünbaum. "Orthogonal Matrix Polynomials Satisfying Second Order Differential Equations." International Math. Research Notices 2004:10 (2004), 461484.

[Durán and Grünbaum 05a] A. J. Durán and F. A. Grünbaum. "Structural Formulas for Orthogonal Matrix Polynomials Satisfying Second Order Differential Equations, I." Constr. Approx. 22 (2005), 255-271.

[Durán and Grünbaum 05b] A. J. Durán and F. A. Grünbaum. "Orthogonal Matrix Polynomials, Scalar Type Rodrigues's Formulas and Pearson Matrix Equations." $J$. Approx. Th. 134 (2005), 267-280.

[Durán and Grünbaum 05c] A. J. Durán and F. A. Grünbaum. "A Characterization of a Class of Weight Matrices with Orthogonal Matrix Polynomials Satisfying Second Order Differential Equations." International Math. Research Notices 2005:23 (2005), 1371-1390.
[Durán and Grünbaum 07] A. J. Durán and F. A. Grünbaum. "Matrix Orthogonal Polynomials Satisfying Second Order Differential Equations: Coping without Help from Group Representation Theory." J. Approx. Theory 148 (2007), 3548.

[Durán and Iglesia 08] A. J. Durán and M. D. de la Iglesia. "Some Examples of Orthogonal Matrix Polynomials Satisfying Odd Order Differential Equations." J. Approx. Theory 150 (2008), 153-174.

[Durán and López-Rodríguez 07] A. J. Durán and P. LópezRodríguez. "Structural Formulas for Orthogonal Matrix Polynomials Satisfying Second Order Differential Equations, II." Constr. Approx. 26 (2007), 29-47.

[Gantmacher 60] F. R. Gantmacher. The Theory of Matrices. New York: Chelsea, 1960.

[Grünbaum 03] F. A. Grünbaum. "Matrix Valued Jacobi Polynomials." Bull. Sciences Math. 127, (2003) 207214.

[Grünbaum and Haine 97] F. A. Grünbaum and L. Haine. "Bispectral Darboux Transformations: An Extension of the Krall Polynomials." International Math. Research Notices 8 (1997), 359-392.

[Grünbaum et al. 03] F. A. Grünbaum, I. Pacharoni, and J. Tirao. "Matrix Valued Orthogonal Polynomials of the Jacobi Type." Indag. Mathem. 14 (2003), 353-366.

[[Krein 49a]] M. G. Krein. "Fundamental aspects of the Representation Theory of Hermitian Operators with Deficiency Index (m, m)." Ukrain. Math. Zh. 1 (1949), 3-66; Amer. Math. Soc. Transl. (2) 97 (1970), 75-143.

[Krein 49b] M. G. Krein. "Infinite J-Matrices and a Matrix Moment Problem." Dokl. Akad. Nauk SSSR 69 (1949), 125-128.

[Littlejohn and Krall 89] L. L. Littlejohn and A. M. Krall. "Orthogonal Polynomials and Higher Order Singular Sturm-Liouville Systems." Acta Appl. Math. 17 (1989), 99-170.

Antonio J. Durán, Departamento de Análisis Matemático, Universidad de Sevilla, Apdo (P.O. Box) 1160. 41080 Sevilla, Spain (duran@us.es)

Received August 6, 2009; accepted April 18, 2010. 